\title{
Reducing Global Warming through Advanced Vehicle Design
}

\author{
R.L. Evans \\ Department of Mechanical Engineering \\ The University of British Columbia, \\ Vancouver, B.C., CANADA \\ evans@mech.ubc.ca
}

\begin{abstract}
Global warming has been identified as one of the most important problems facing mankind in the 21st century. Currently, some 6 gigatonnes of $\mathrm{CO} 2$ are emitted each year as a result of the combustion of fossil fuels, and a large fraction of these emissions originate from the transportation sector. By examining the complete energy conversion chain, the choice of primary energy source for any particular application becomes easier to understand. A discussion of alternatives to the internal combustion engine as the sole power source for vehicular propulsion is presented, and some form of hybrid electric vehicle propulsion system is identified as being a likely choice to reduce fossil fuel consumption, and therefore $\mathrm{CO}_{2}$ emissions from the transportation sector. The demonstrated market success of grid-independent hybrid vehicles may be followed by a new design of "plug-in hybrid" vehicles in which it is possible to travel for up to $100 \mathrm{~km}$ in an all-electric mode, while maintaining the option of using an internal combustion engine when greater range between charging cycles is required.
\end{abstract}

\section{Introduction}

The provision of clean, and sustainable, energy supplies to satisfy our ever-growing energy needs is one of the most critical challenges facing mankind at the beginning of the $21^{\text {st }}$ century. The problem is made even more acute by the large and rapidly growing appetite for energy in the developing world, many of which are experiencing extremely high economic growth rates, leading to equally high demands for new energy supplies. The growing global demand for energy in all of its forms is naturally putting pressure on the declining supplies of traditional fossil fuels, particularly crude oil and natural gas. However, in recent years no major new production fields have been found, and the exploration effort and cost required to maintain these ratios has been significantly increased. Ultimately, of course, supplies of oil and natural gas will be depleted to such an extent, or the cost of production will become so high, that alternative energy sources will need to be developed. Global climate change, in particular the prospect for global warming, has put the spotlight on our large appetite for fossil fuels. Although there is considerable debate on the extent of the problem, there is little doubt that the atmospheric concentration of $\mathrm{CO}_{2}$, one of the key "greenhouse gases", is increasing quite rapidly, and that this is likely due to mankind's activities on earth, or "anthropogenic" causes. The utilization of any fossil fuel results in the production of large quantities of $\mathrm{CO}_{2}$, and most scientific evidence points to this as the main cause of increasing concentration levels in the atmosphere. We must, therefore, develop new long-term methods of strategic thinking and planning, and make sure that some of the best minds, with a wide-range of skills and abilities, are given the tools to do the job. This paper describes one design approach to solving the transportation energy problem through the design and development of gridconnected electric vehicles.

\section{The Energy Conversion Chain}

Every time we use energy, whether it's to heat our home, or fuel our car, we are converting one form of energy into another form, or into useful work. When we drive a car we are using the engine to convert the chemical energy in the gasoline into mechanical work to power the wheels. This is just one example of the "Energy Conversion Chain" which is always at work when we use energy. In each case we can trace a source of "primary energy" through several conversion steps into the final end-use form, such as thermal energy for space heating or mechanical work to power a motor vehicle. Unfortunately, many proposals to change the 
ways in which we supply and use energy take only a partial view of the energy conversion chain, and do not consider the effects, or the costs, that the proposed changes would have on the complete energy supply system. A schematic of a generic "energy conversion chain" is shown in Figure 1. The chain starts with "primary" energy sources, of which there are only three; fossil fuels, renewable energy, and nuclear power. The chain also ends with just three major end-use categories; transportation, commercial and domestic buildings, an industrial energy use. After processing, the primary energy sources end up as energy "carriers", which include refined petroleum products and electricity. Hydrogen, which is sometimes promoted as a fuel of the future through the so-called "hydrogen economy", is not an energy source at all, but only a potential "energy carrier". In some cases, not all steps in the chain are required, but energy end-use can always be traced back to a primary energy source. For example, in most cases when electricity is the energy carrier it is used immediately upon production, due at least in part to the difficulty of storing electricity.

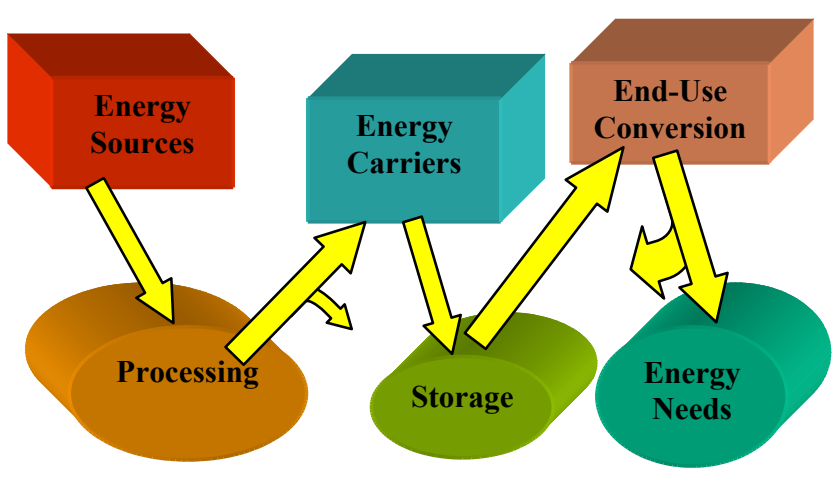

Figure 1. The Energy Conversion Chain

An important feature of the energy conversion chain is the release of emissions, both in the initial processing step and in the final end-use conversion step. Some of these are released in the refining process, but most of them are released during the final conversion from chemical energy to useful work in the vehicle's engine. This emission of pollutants from both the primary energy processing step, and the end-use step, provides an extremely important link between energy use and the environment. The reaction of unburned hydrocarbons and $\mathrm{NO}_{\mathrm{x}}$, in the presence of sunlight, for example, is responsible for smog formation, which has become a major problem in urban centres. This has been alleviated somewhat in the developed world by the introduction of stringent regulations to limit emissions from vehicles and power stations, but will continue to be a very serious problem with the growth in vehicle ownership, particularly in large developing economies. The transportation sector continues, however, to be a major source of greenhouse gas emissions, and the search for a viable source of non-fossil fuel energy for the transportation sector is one of the most important scientific and engineering challenges of the $21^{\text {st }}$ century.

\section{Non-Fossil Fuel Energy for Transportation Applications}

Liquid petroleum fuels are ideally suited to transportation applications because of their inherently high energy density, and the ease of transportation and storage of these fuels. The internal combustion engine has reached a high level of development, and this is now almost universally used as the power source for all road vehicles. The concern with using petroleum fuels, of course, is that they are derived from crude oil, a nonrenewable resource which will eventually be in very short supply. Also, the combustion process produces emissions of nitrogen oxides, carbon monoxide, and unburned hydrocarbons, as well as large quantities of $\mathrm{CO}_{2}$, the principal greenhouse gas. One way to reduce the dependence of the transportation sector on petroleum based fuels is to design a new form of propulsion system which replaces the internal combustion engine fuelled by petroleum with a completely different form of energy carrier. This has been done successfully for rail transportation by using electric locomotives on lines with heavy traffic volumes. This is possible for rail transportation since electrical power can be provided continuously to the locomotive through overhead electrical cables, or through a "third-rail" placed adjacent to the tracks. Although this provides a very clean source of energy at the point of end-use, if the electricity is generated primarily from fossil fuels, then there may be no net reduction of greenhouse gas emissions as a result of railway electrification. If, in the long term, the electricity "carrier" is generated primarily from nonfossil fuel sources, such as renewable energy or nuclear power, then there will be a direct benefit through the elimination of greenhouse gas production from the railways. For road vehicles, however, it is not practical to provide electrical power continuously to cars or trucks, and purely electric vehicles must rely on energy stored in an on-board battery. Although electric cars were common during the very early development of motor vehicles, the low energy capacity of batteries made them uncompetitive with vehicles powered by internal combustion engines, and they disappeared from the marketplace. 
Proponents of the "hydrogen economy" claim that the use of hydrogen as a transportation fuel would eliminate the production of any harmful exhaust emissions from vehicles on the road. This is, of course, true for the vehicle itself, but as we have seen in our discussion of the complete energy conversion chain, it only represents one part of the complete energy use picture. Hydrogen would just be an energy carrier, like gasoline or electricity is today, and it would need to be "manufactured" from one of the three primary energy sources. If this primary source were to be a hydrocarbon fuel, such as natural gas or coal, all of the carbon in the primary energy source would still end up as $\mathrm{CO}_{2}$ at the point of hydrogen production. If, on the other hand, the hydrogen produced from a more sustainable primary energy source, such as renewable energy or nuclear power, then there would indeed be no production of greenhouse gases anywhere in the energy conversion chain. The energy conversion chain for a vehicle design using hydrogen in this manner is illustrated by the schematic diagram in Figure 2. This shows the primary energy source as some form of renewable energy, represented by PV solar cells generating electricity in the figure, but this could be wind-power, or any other source of renewable energy or nuclear power. Following along the energy conversion chain, the electricity would then be used to produce hydrogen by electrolysis of water, and the hydrogen would then be compressed, or converted into liquid form, for storage on board the vehicle. The vehicle would utilize all-electric drive, and a fuel cell would be used to generate electricity on-demand from the hydrogen, which would then be supplied to an electric motor providing the mechanical power to drive the vehicle.

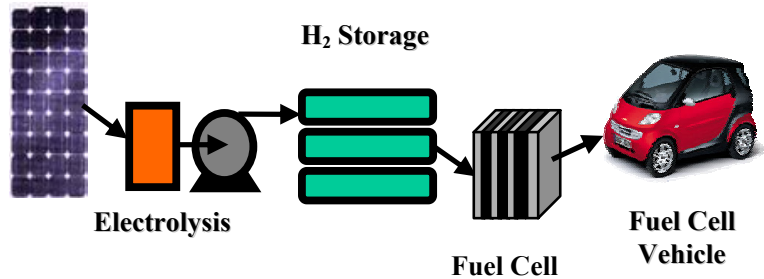

Figure 2. Energy Conversion Chain for a Fuel
Cell Vehicle

We can see from Figure 2, however, that the fuel cell is just one major component of the hydrogen fuelcell powered automobile. A very critical component of the vehicle propulsion system is the fuel storage system on board the vehicle. For a conventional motor vehicle, utilizing an internal combustion engine, this is the simple fuel tank, which stores either gasoline or diesel fuel, both of which conveniently exist as liquid fuels at normal ambient temperature and pressure conditions. At these same ambient temperature and pressure conditions, however, hydrogen is a gas, and this gas has a very low energy density. In order to carry a significant quantity of energy on-board the vehicle in the form of hydrogen, therefore, it would need to be highly compressed, or perhaps even liquefied and stored in a "cryogenic" fuel tank at a temperature of around $250^{\circ} \mathrm{C}$. In order to store enough hydrogen energy to provide a reasonable driving range, engineers have proposed using compressed hydrogen at a pressure of 350 bar (5,000 psi), or even 700 bar (10,000 psi). These very high pressures require heavy gas storage cylinders, which would add considerable weight and volume to the vehicle compared to the usual sheet metal container used for liquid fuels. In fact, the design of hydrogen storage systems for vehicles is one of the most difficult challenges facing the successful commercialization of hydrogen fuelled vehicles. There is also a substantial loss of available energy from both the electrolysis process and the compression of hydrogen for highpressure storage.

One of the advantages claimed for fuel cell vehicles is the much higher energy conversion efficiency of fuel cells, compared to internal combustion engines. The efficiency of a fuel cell, at around $50 \%$, is certainly much higher than can be expected for the typical internal combustion engine used in motor vehicles. However, if the hydrogen used as the energy carrier on board the vehicle were to be derived from fossil fuels, as it will almost certainly be in any early stage of commercialization, then the overall "well-to-wheels" efficiency is unlikely to be significantly higher than that of the best available technology using a conventional internal combustion engine. This is the conclusion found in comparative studies of vehicle powertrain efficiency by both the Argonne National Laboratory of the U.S. Department of Energy [1], and by researchers at the Massachusetts Institute of Technology [2]. For both of these studies the hydrogen was assumed to be obtained from a refuelling station by reforming natural gas, which would be the most likely source of primary energy, at least in the early phase of fuel cell commercialization. Also, if the primary energy source was a fossil fuel, as assumed in the study, the result would be almost identical levels of $\mathrm{CO}_{2}$ emissions. It seems unlikely, therefore, that this small gain in overall vehicle efficiency would be sufficient to overcome the much higher cost and complexity of the hydrogen storage system and the fuel cell itself. Advocates of fuel cell vehicles, however, contend that in the long term hydrogen will be produced from some form of renewable energy, as illustrated in Figure 2, or perhaps 
from nuclear power, which would then result in no emissions of $\mathrm{CO}_{2}$ for the complete energy conversion chain.

If we examine the case in which hydrogen is generated from a sustainable primary resource, as illustrated in Figure 2, then the first step in the energy conversion chain is the generation of electricity as an initial energy carrier. This carrier is then converted into hydrogen as a secondary carrier, and this is stored onboard the vehicle. The final step in the chain is then the conversion of the stored hydrogen back into electricity by the fuel-cell, and this is then used to power the electric propulsion motor. A parallel situation is used in a simple battery electric vehicle, in which a battery is used on-board the vehicle to store the electricity, as shown by the simple energy conversion chain schematic in Figure 3. In this case there is no need to convert the electricity into a secondary carrier, since the electricity generated as the primary carried is stored directly by the battery, and is then used when needed to supply the electric propulsion motor. The only difference between the scenarios depicted in Figure 2 for the fuel-cell vehicle, and in Figure 3 for the battery electric vehicle, is that in the first case energy is stored in the form of hydrogen, and in the second case in the form of electrical energy in the battery.

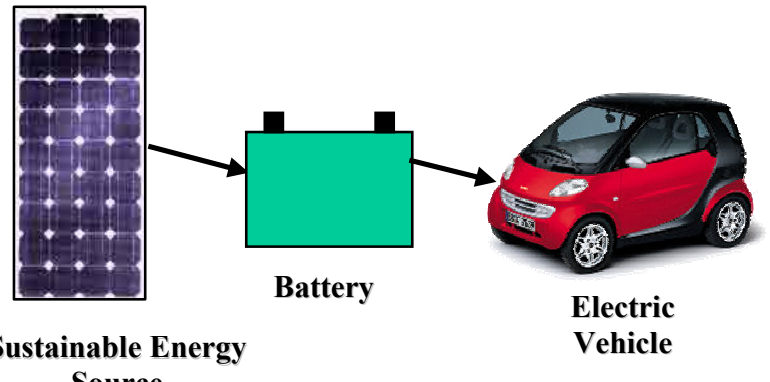

Source

\section{Figure 3. Energy Conversion Chain for a Battery Electric Vehicle}

The difference in these two different design approaches can then be summarized graphically as illustrated in Figure 4. This shows the energy conversion chain for the two different approaches, starting from the point at which the primary energy source produces electricity, and ending where electricity is again used to power the vehicle's electric motor. In other words, all of the equipment illustrated by the conversion chain in the top half of the figure, consisting of hydrogen production by electrolysis, compression, storage in high-pressure cylinders, and finally conversion back into electricity by a fuel cell, is directly analogous to the electrical battery in the lower half of the figure. By making a simple comparison it can be clearly seen that all of the equipment required for the fuel cell vehicle, including hydrogen production, storage, and the fuel cell, is really just an electrical energy storage device.

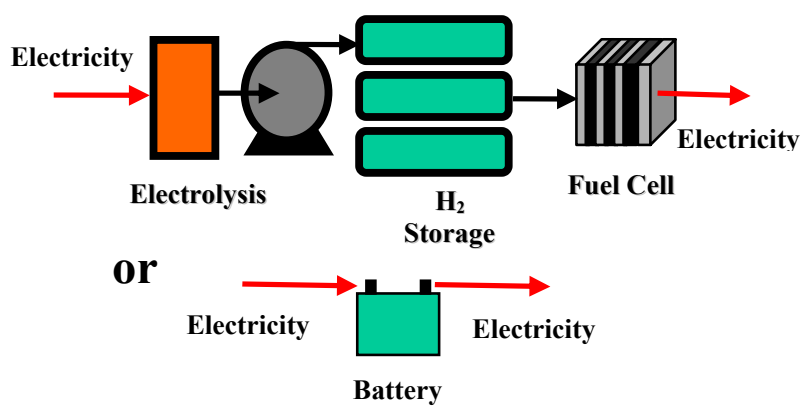

Figure 4. Alternative Electrical Energy Storage Concepts

Although strictly speaking not all of the process steps shown in Figure 4 are energy conversion processes, there is a loss of available energy associated with each step. For example, since it takes about $8 \%$ of the total energy content of the hydrogen to compress it to the pressure (200 Bar) used in the storage cylinders, for every $100 \mathrm{~kJ}$ of hydrogen energy produced by electrolysis, the net energy that then resides in the hydrogen storage is around $92 \mathrm{~kJ}$. To account for these energy losses we may assign an "in-out" efficiency value to each step in the process. For the battery, there is only one step between the electrical input to the energy storage and energy output to the vehicle, and since about $10 \%$ of this energy is normally lost in the form of heat during the battery charging process, we can assign an "in-out efficiency" of $90 \%$ to the battery.

Table 1. "In-Out” Energy Efficiency

\begin{tabular}{|l|c|c|c|}
\hline \multicolumn{2}{|c|}{ Fuel Cell } & \multicolumn{2}{c|}{ Battery } \\
\hline \hline Electrolysis & $65 \%$ & Battery & $90 \%$ \\
\hline Compression & $92 \%$ & - & - \\
\hline Fuel Cell & $50 \%$ & - & - \\
\hline Overall Efficiency & $\mathbf{3 0 \%}$ & Overall Efficiency & $\mathbf{9 0 \%}$ \\
\hline
\end{tabular}

The "in-out" efficiencies for each of the steps in the two equivalent fuel cell "storage" processes of Figure 4 are shown in Table 1 for comparison. The efficiencies of each individual step are then multiplied together to get the final "overall efficiency" of the complete process, going from electricity "in" from the primary source to electricity "out" to the traction motor. 
With an assumed fuel cell energy conversion efficiency of $50 \%$, the overall "in-out efficiency" for the fuel cell conversion chain shown in Figure 4 is just $30 \%$, compared to $90 \%$ for the conventional battery. In other words, if we were going to use electricity from some sustainable primary energy source as the energy carrier for a vehicle, then for every $100 \mathrm{~kJ}$ of primary energy used, we would "deliver" some $90 \mathrm{~kJ}$ to the vehicle's electric traction motor using a battery for energy storage. If, however, we used hydrogen and a fuel cell as the energy "storage" system, for every $100 \mathrm{~kJ}$ of primary energy used, only some $30 \mathrm{~kJ}$ would be delivered to the traction motor. We have used an estimate of the current efficiency of electrolytic production of hydrogen from water of around $65 \%$, although research and development is under way to significantly raise this value. Even if the efficiency of electrolysis were to be increased to $80 \%$, however, the overall "in-out" efficiency would still only reach $37 \%$. This simple analysis indicates that if a battery with sufficient energy storage density and specific energy to provide a reasonable vehicle range were available, this would be a very attractive design alternative to the hydrogen and fuel cell route.

If electrical batteries were able to store sufficient energy to provide a range of up to 100 miles, then relatively simple battery electric vehicles, which would normally be re-charged overnight, or when not in use for several hours, would likely be attractive to most consumers. Such vehicles would be much less complex and likely much cheaper to produce than the comparable fuel cell vehicles together with the necessary hydrogen production and storage systems. Batteries are not yet able to compete with liquid fuels in terms of either energy density or specific energy, and pure battery electric vehicles will likely be suitable only for specialized short range applications in the near-term. There continues to be development work on batteries, however, and this has been driven primarily by the successful introduction in the last few years of hybrid electric vehicles (or HEVs). These use a propulsion system consisting of a conventional internal combustion engine, acting as the "prime mover", in parallel with an electric motor and storage battery. All of the energy to drive the vehicle still comes from the liquid fuel (gasoline or diesel fuel) used by the internal combustion engine, but the engine is used to either charge the battery via a generator, or to drive the wheels directly as in a conventional vehicle, or in some combination of both of these approaches. During low-speed operation, and particularly in stop-and-go driving in urban centres, the engine is shut down, and all propulsion is provided by the electric motor being fed from the battery. As the battery becomes discharged the engine is automatically started and again begins to charge the battery, and may also provide some mechanical propulsion directly to the wheels through a gearbox. One major benefit of this powertrain design is that the engine can now operate at its most efficient design condition, independently of vehicle speed or load, thus greatly increasing the overall fuel efficiency. Another significant feature of hybrid vehicles is the use of "regenerative braking", which utilizes the generator to absorb much of the braking energy normally dissipated in the form of heat by the brakes, and then uses this energy to re-charge the battery. As a result of these design features a hybrid vehicle normally has lower fuel consumption during city driving than on the highway, making them particularly well suited to urban commuting. These two features have been developed and refined by automotive engineers so that the hybrid vehicle has a fuel efficiency about $50 \%$ greater than that of a conventional vehicle powered by an internal combustion engine alone.

The current design of hybrid vehicles may be classed as "stand-alone", or "grid-independent" hybrids, because although they incorporate an electrical powertrain, and storage battery, they obtain all of their primary energy from the fuel carried on-board the vehicle, and do not need to be plugged in to the electrical grid to re-charge the battery. However, with the expected advances in battery energy density, and the desire to minimize the use of fossil fuels in vehicles, they may very well be the precursor to a transition to the next generation of hybrid vehicles; the so-called "plugin" hybrids, sometimes also referred to as "gridconnected hybrids". A simple schematic of this concept, which incorporates the advantages of both battery electric vehicles and hybrid vehicles, is shown in Figure 5.

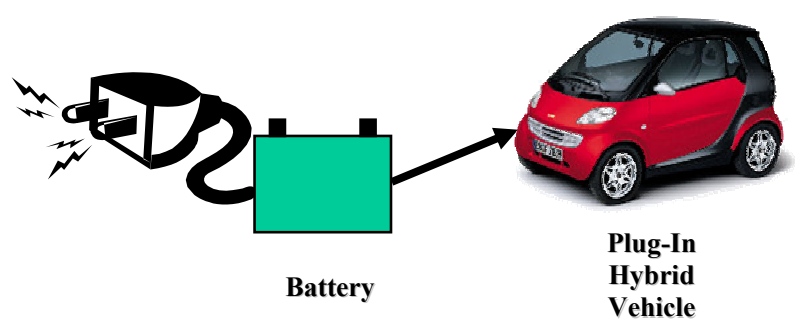

Figure 5. The "Plug-in Hybrid" Electric Vehicle

In this design concept, the battery pack in an otherwise conventional hybrid vehicle would be much larger, and could be fully charged when not in use by being plugged into the electrical grid. The vehicle could operate for a significant range, perhaps somewhere between 20 and 60 miles, as a completely electric 
vehicle, but would still have a small engine to re-charge the battery only when necessary in order to exceed the "all-electric" range or perhaps when climbing very steep hills. For many drivers, and certainly for most commuters, the vehicle would then be capable of operating as a pure battery electric vehicle for most trips, and would be plugged in overnight and perhaps also when not in use during the working day. The successful development and introduction into the marketplace of the "plug-in" hybrid vehicle would mark the beginning of a significant new transportation design paradigm, that of disconnecting road vehicles from the need to use petroleum fuels, at least for the majority of kilometres travelled. In considering the complete energy conversion chain for this option, if electricity were to be generated primarily by sustainable primary energy sources, such as renewable energy or nuclear power, then road transportation would also become much more sustainable and would no longer be a significant factor in contributing to greenhouse gas production.

The Electric Power Research Institute (EPRI) in the U.S. has recently published the results of a study [3] comparing the performance of plug-in hybrid electric vehicles to a stand-alone hybrid vehicle, (or HEV 0) and a conventional vehicle powered by a gasoline engine. Two plug-in hybrid vehicle designs were considered, one with an all-electric range of 20 miles (HEV 20) and one with an all-electric range of 60 miles (HEV 60). All of the HEVs were assumed to use state-of-the-art nickel-metal hydride (NiMH) batteries and regenerative braking, and to have similar performance, including a minimum top speed of $90 \mathrm{mph}$ and a $0-60 \mathrm{mph}$ acceleration time of less than 9.5 seconds. All vehicles were assumed to have sufficient gasoline storage to provide a range of 350 miles. The power split between engine and electric motor is approximately equal for the HEV 0 and the HEV 20, while for the HEV 60 the electric motor has about twice the power of the gasoline engine. In considering the overall energy consumption of all of the vehicles the study took a "well-to-wheels" approach, and included the energy obtained from the gasoline on-board the vehicle and the energy required to process the crude oil to produce the gasoline, as well as the electrical energy required to re-charge the batteries for both of the plug-in hybrid vehicles. For the battery re-charging part of the process, the study assumed that electricity would be generated from natural gas using a combined cycle power plant, with an overall thermal efficiency of approximately 50\%. Many different performance parameters were calculated during the simulation over a standard driving cycle, but the main results of the study were that the complete "well to wheels" $\mathrm{CO}_{2}$ emissions for the HEV 20 and the HEV 60 were approximately one-half, and one-third, respectively, of that for the conventional vehicle. If, on the other hand, the electricity supply was assumed to be from a sustainable source, with no $\mathrm{CO}_{2}$ emissions, then the total well-to-wheels $\mathrm{CO}_{2}$ emissions over the driving cycle, compared to those of a conventional vehicle, would be reduced by two-thirds for the HEV 20, and by some $87 \%$ for the HEV 60 vehicle.

\section{Conclusions}

The need to move from the use of fossil fuels for nearly all transportation applications to a more sustainable energy source will be one of the most important design challenges facing engineers in the $21^{\text {st }}$ century. When the complete energy conversion chain for motor vehicle transportation is examined, the use of hydrogen as a carrier does not appear to be particularly attractive. Only if the hydrogen is made by electrolysis using electricity from sustainable primary resources, such as renewable energy or nuclear power, will there no production of greenhouse gases. This scenario implies, however, that electricity will be used first to produce a secondary carrier, hydrogen, and then this carrier will be returned to electricity on-board the vehicle, using a fuel cell. In this case the complete onboard energy storage and end-use conversion system simply acts like a battery, with electricity both coming in and going out. A more attractive scenario appears to be development of the "plug-in", or "grid-connected" hybrid electric vehicle, which uses electricity from the grid to charge a battery rather than to generate hydrogen, but maintains a small fossil-fuelled engine as a "back-up" device. Recent studies have shown that using this approach for vehicle propulsion, in which the battery would provide an "electric-only" range of some 60 miles $(100 \mathrm{~km})$, would result in a reduction of greenhouse gas emissions by up to $87 \%$ compared to those from a conventional fossil-fuelled vehicle.

\section{References}

1. Rousseau, A., et al., Well-to-Wheels Analysis of Advanced SUV Fuel Cell Vehicles, SAE paper 2003-01-0415, 2003.

2. Weiss, M.A., et al., Comparative Assessment of Fuel Cell Cars, MIT LFEE Report 2003-001 RP, 2003.

3. Graham, R., et al., Comparing the Benefits and Impacts of Hybrid Electric Vehicle Options, EPRI Report 1000349, 2001. 